\title{
Hereditary Multiple Exostoses
}

National Cancer Institute

\section{Source}

National Cancer Institute. Hereditary Multiple Exostoses. NCI Thesaurus. Code C5183.

Hereditary disorder transmitted by autosomal dominant genes (EXT1-3) and

characterized by multiple exostoses (multiple osteochondromas) near the ends of long bones. The genetic abnormality results in a defect in the osteoclastic activity at the metaphyseal ends of the bone during the remodeling process in childhood or early adolescence. The metaphyses develop benign, bony outgrowths often capped by cartilage. A small number undergo neoplastic transformation. 